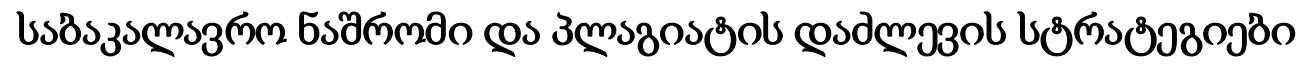

\author{
дsäзs

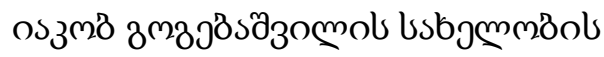

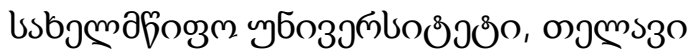

https://doi.org/10.52340/idw .2021.533

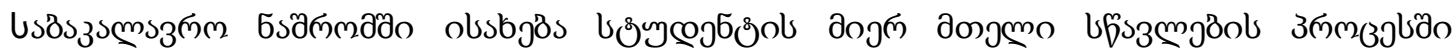

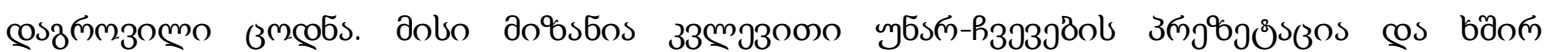

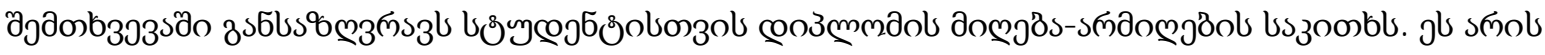

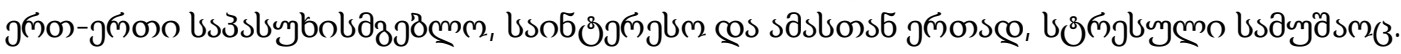

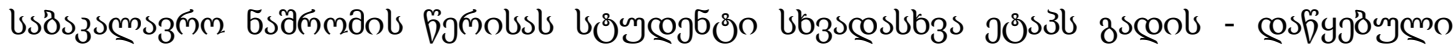

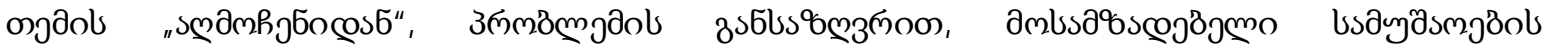

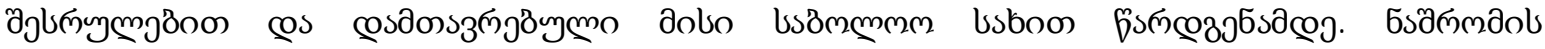

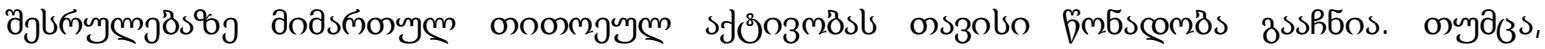

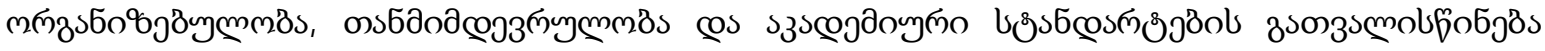

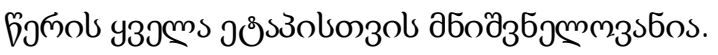

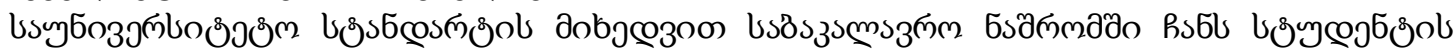

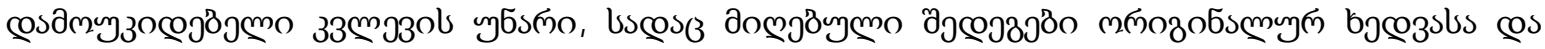

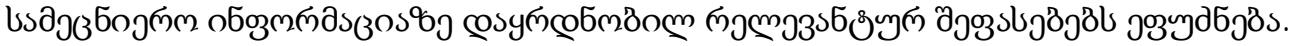

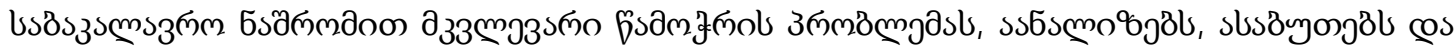

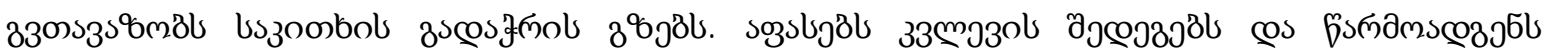

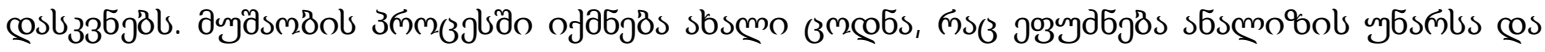

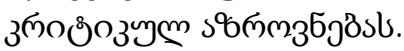

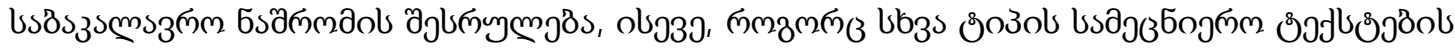

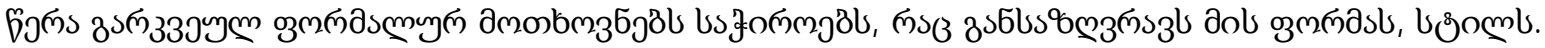

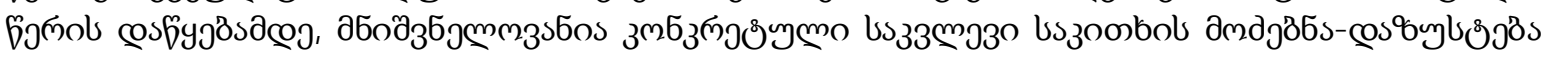

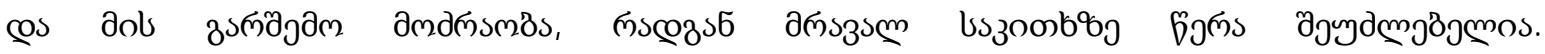

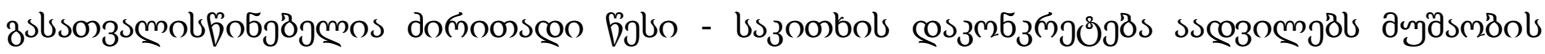
उलмखुjll.

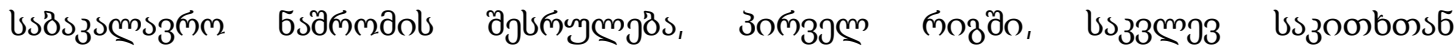

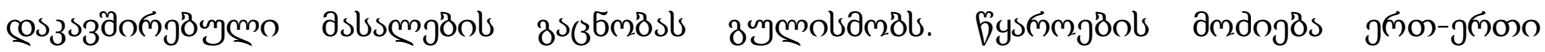

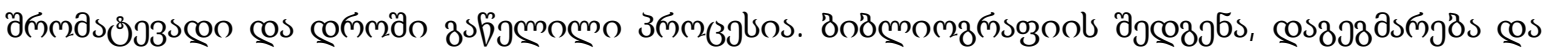

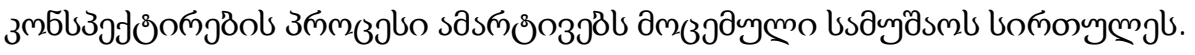

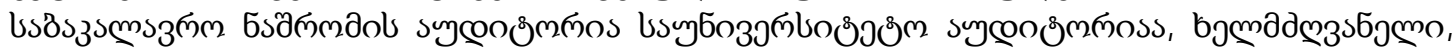

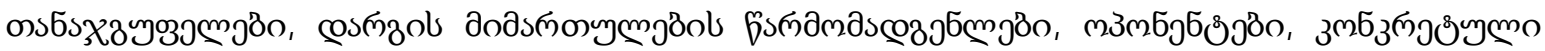

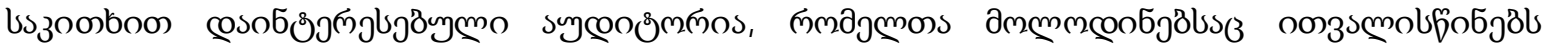
аззмэзรณо.

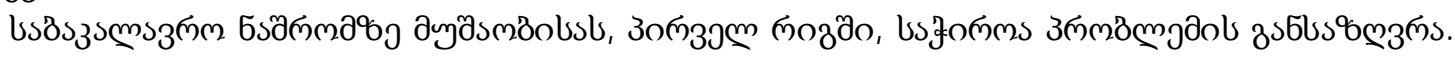

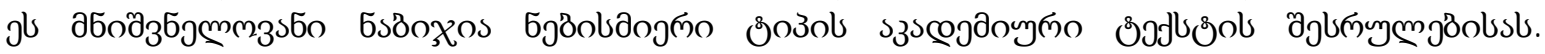

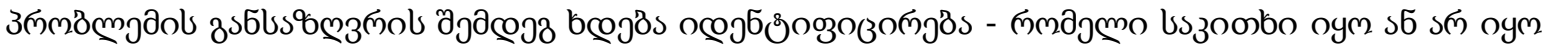

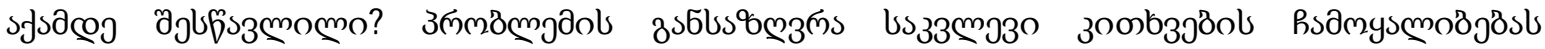

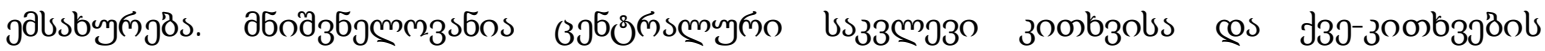

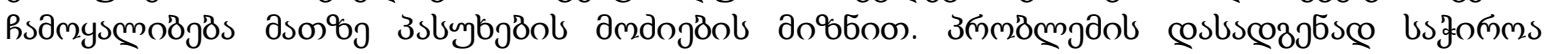




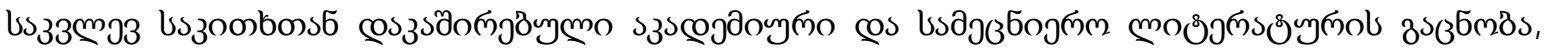

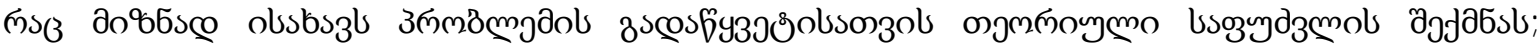

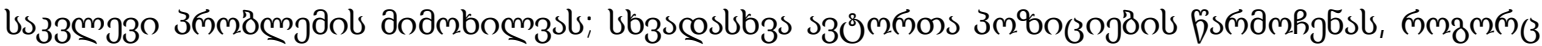

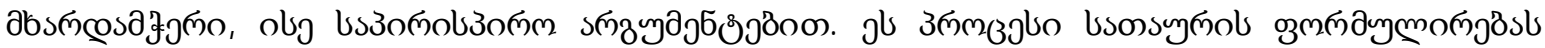

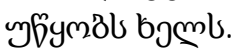

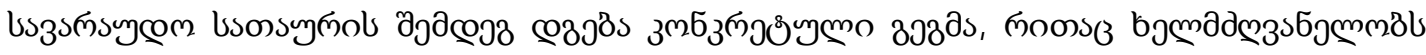

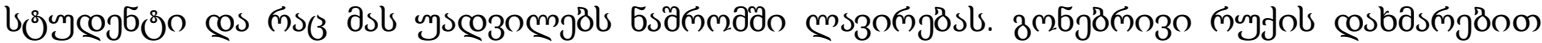

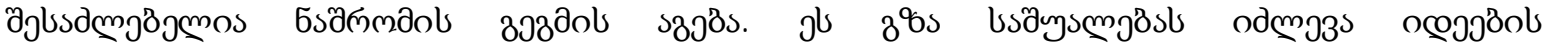

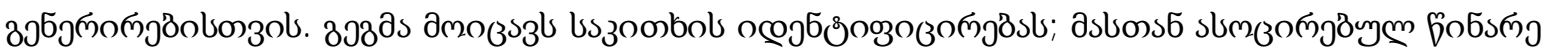

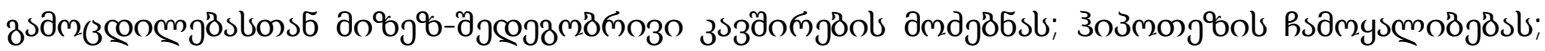

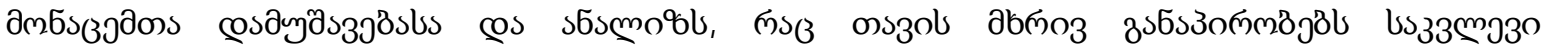

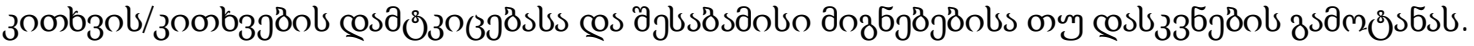

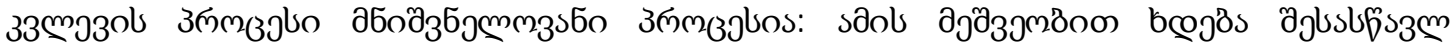

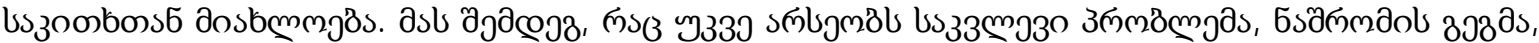

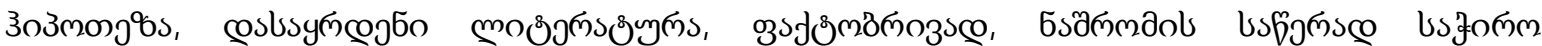

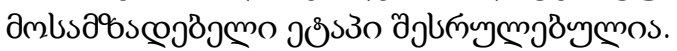

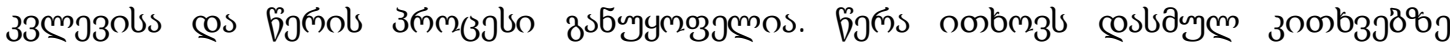

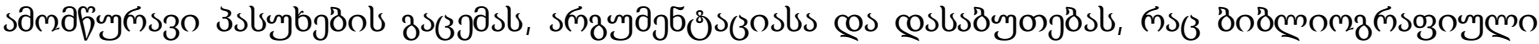

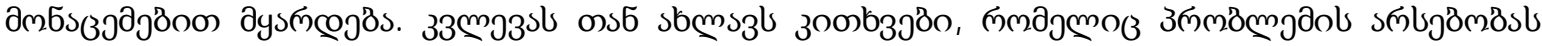
оз

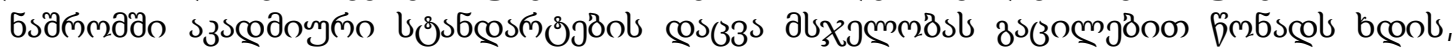

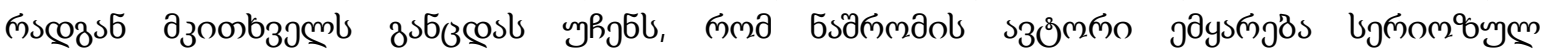

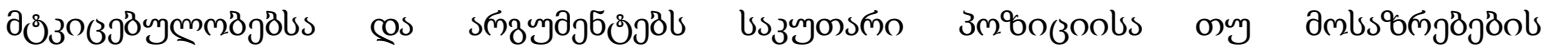

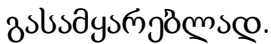

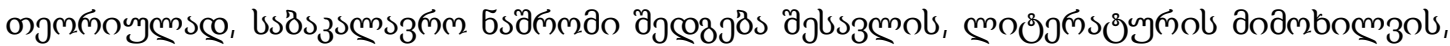

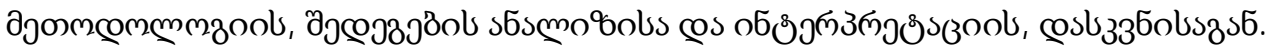

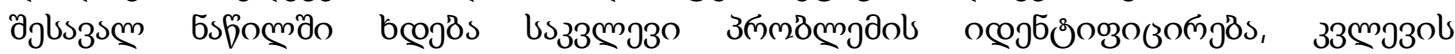

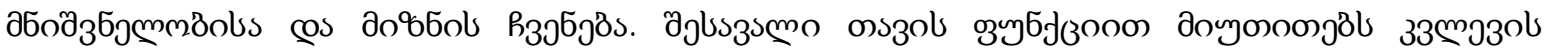

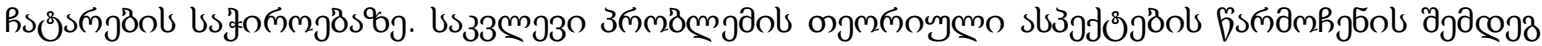

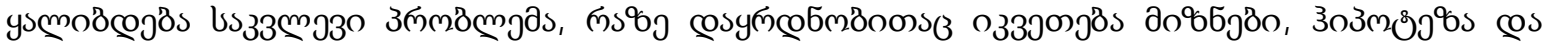
usзm

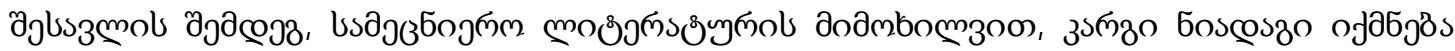

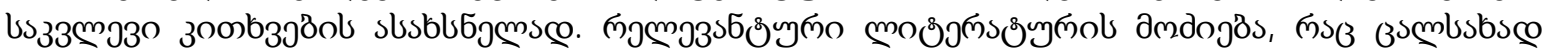

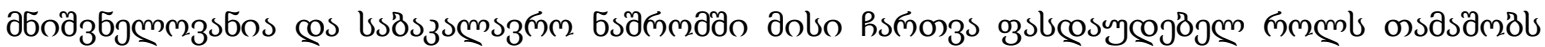

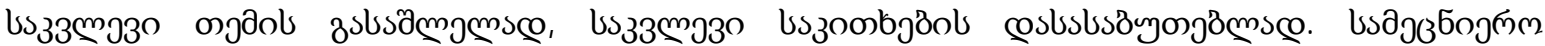

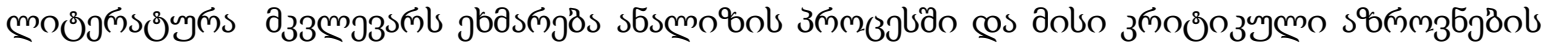

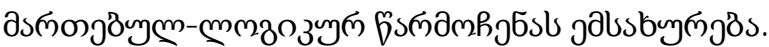

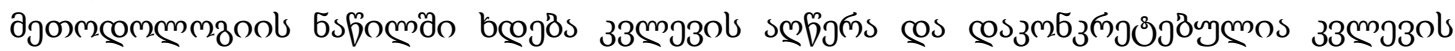

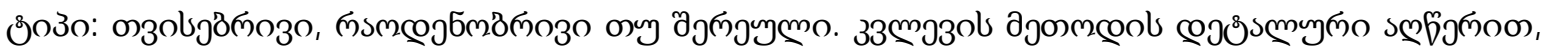

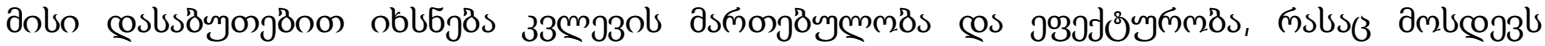

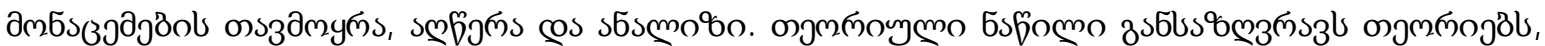

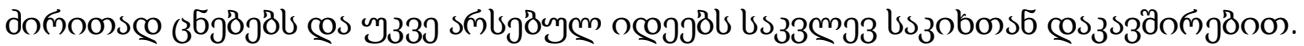

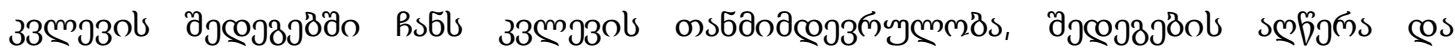

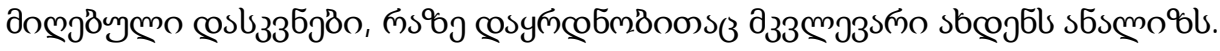

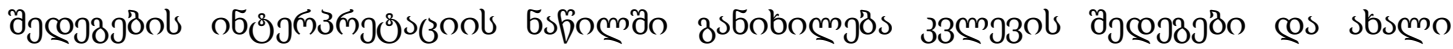

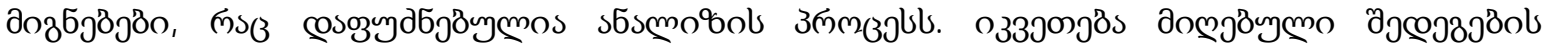

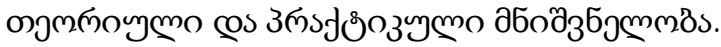

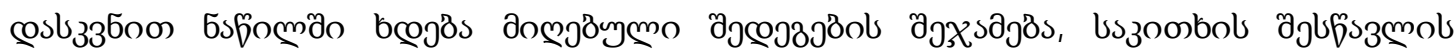

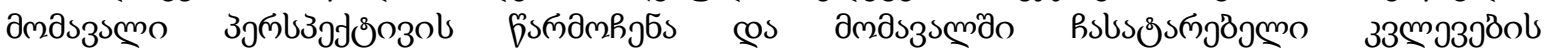

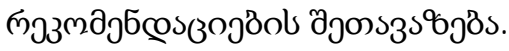




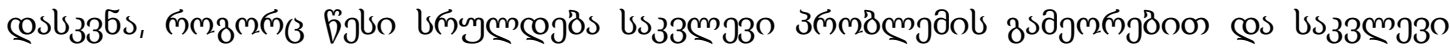

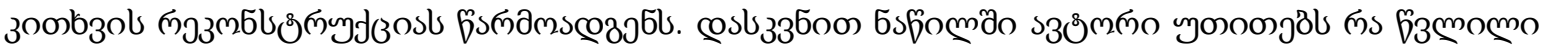

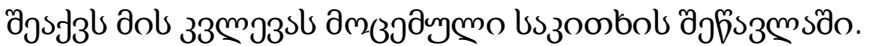

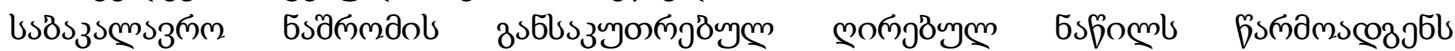

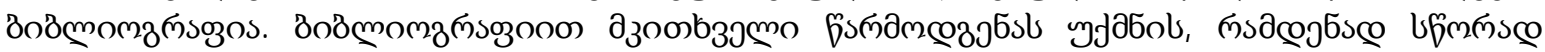

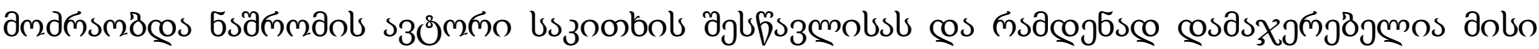

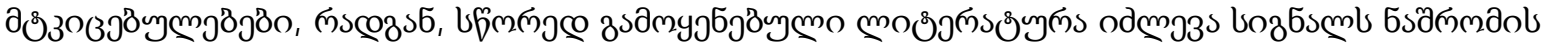

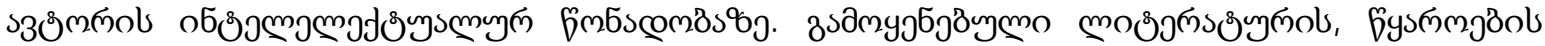

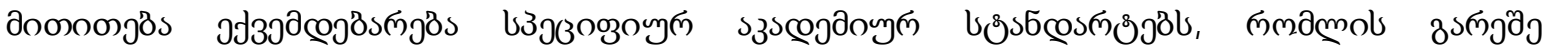

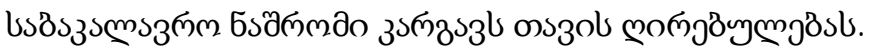

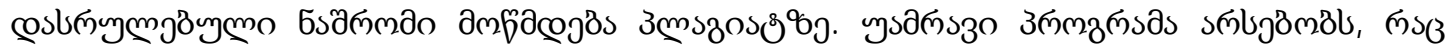

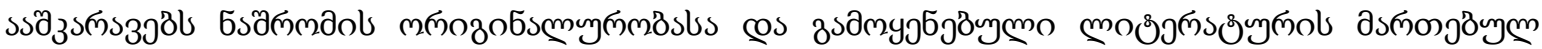

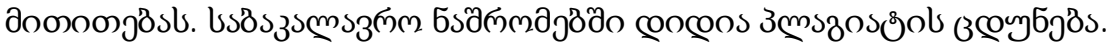

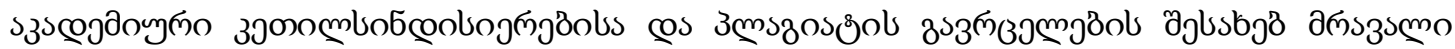

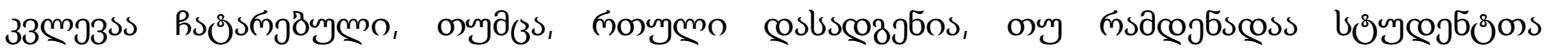

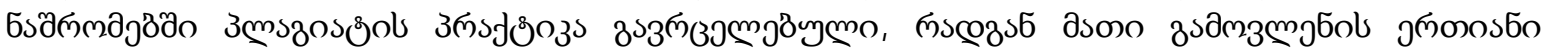

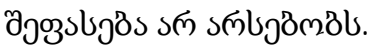

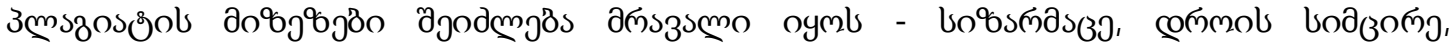
обఠэ

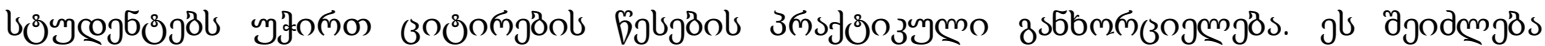

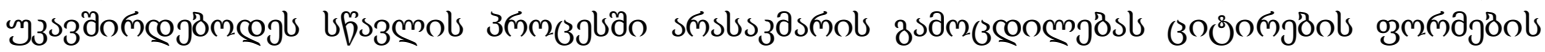

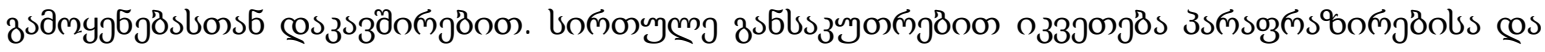

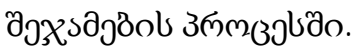

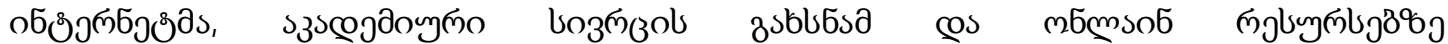

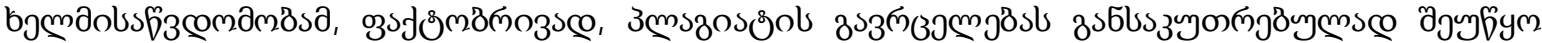

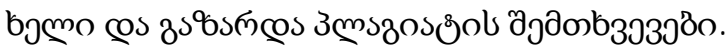

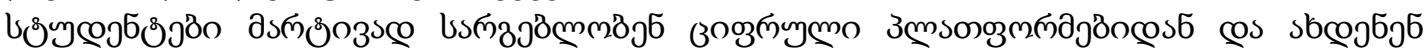

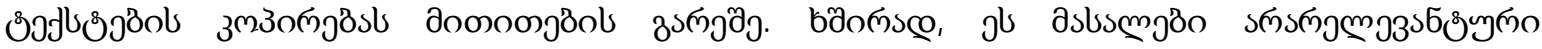

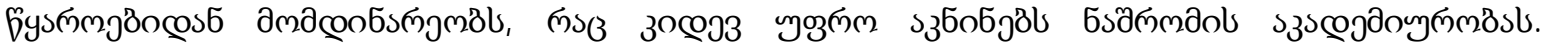

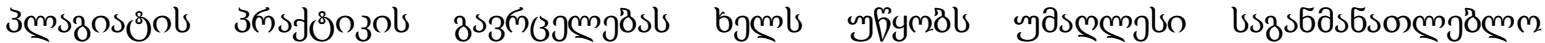

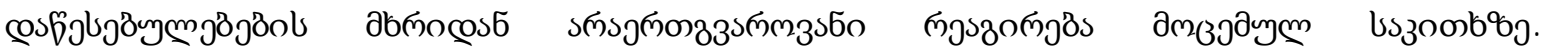

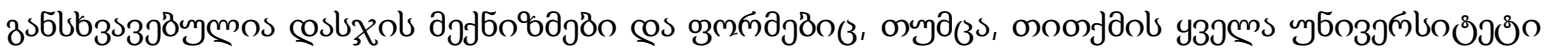

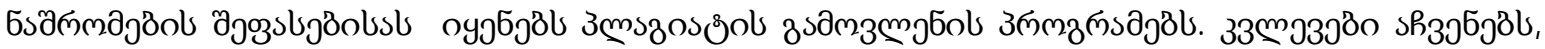

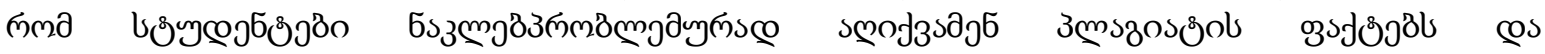

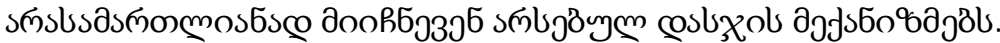

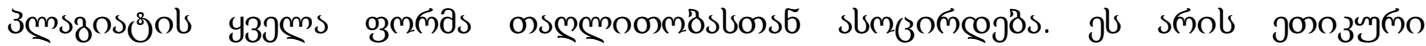

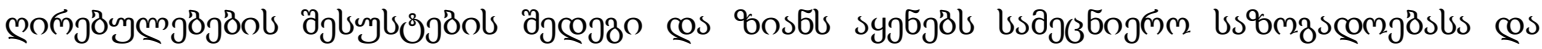

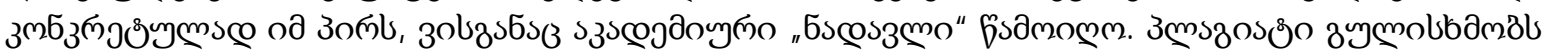

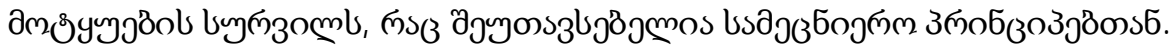

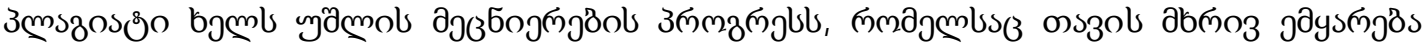

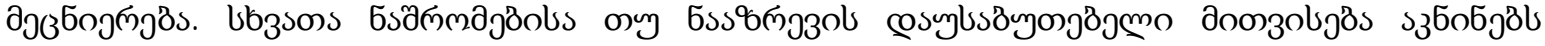

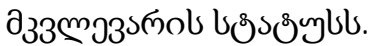

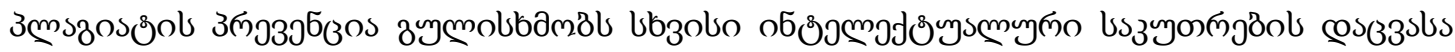

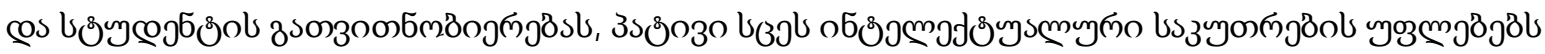

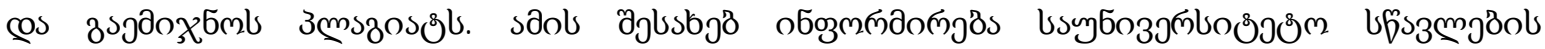

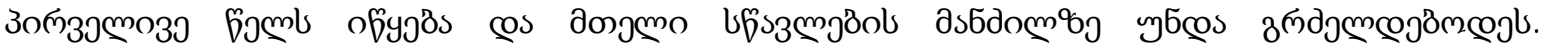

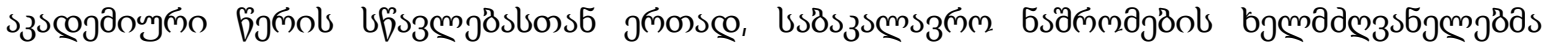

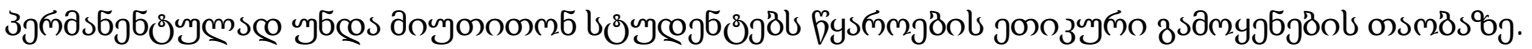

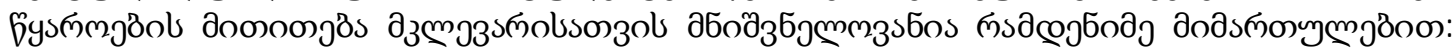

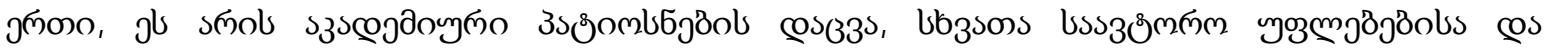




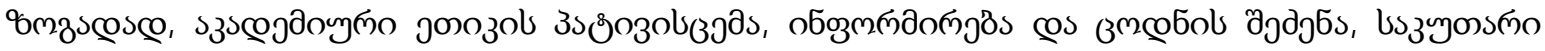

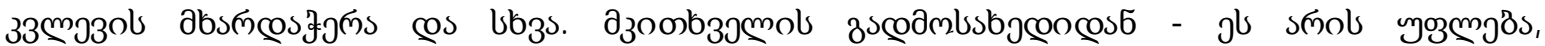

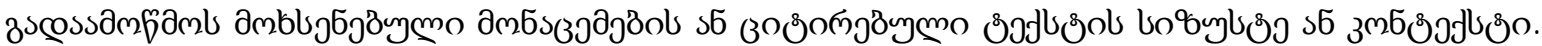

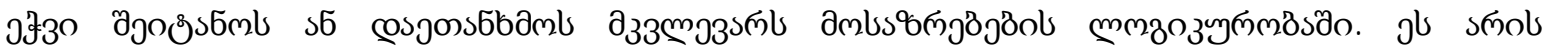

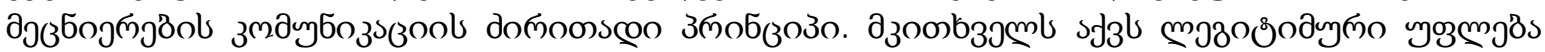

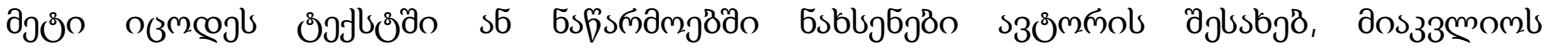

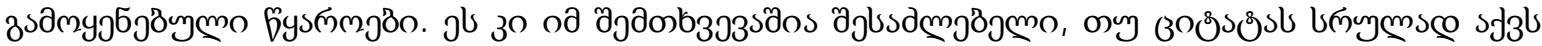

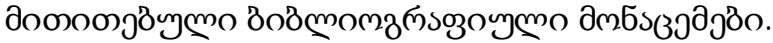

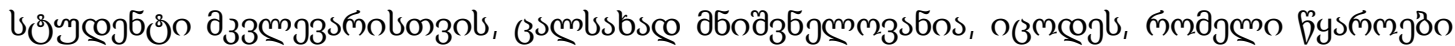

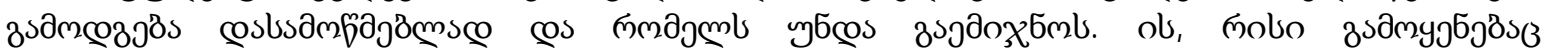

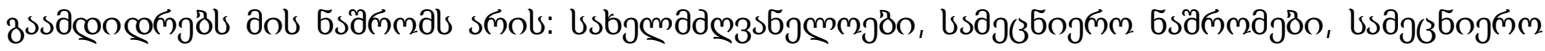

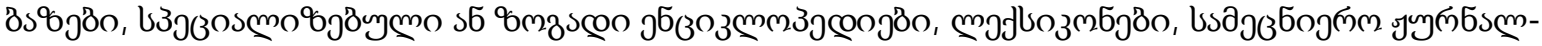

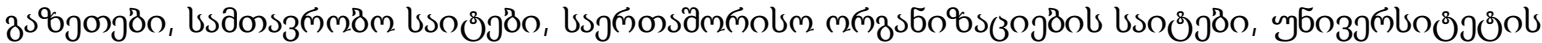
иsоð์

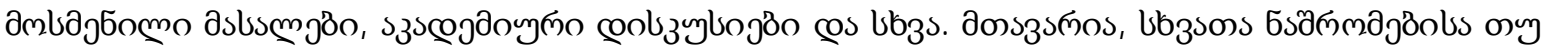

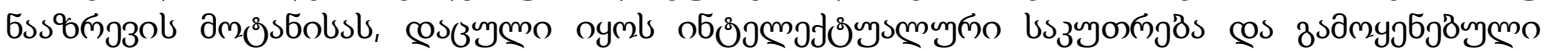

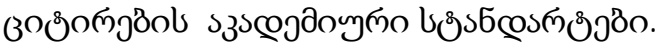

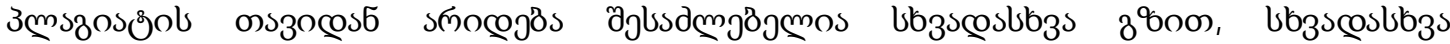

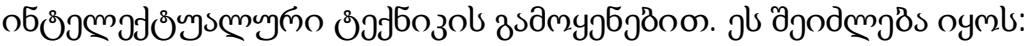

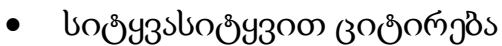

- उsmsognssion

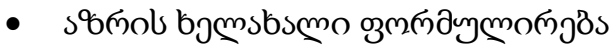

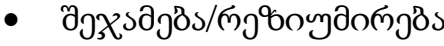

- onsmazsбo

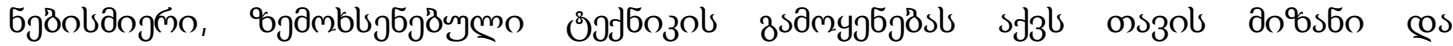

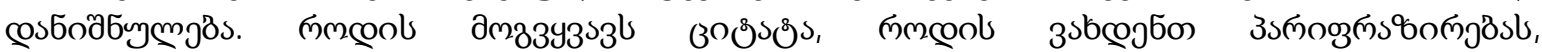

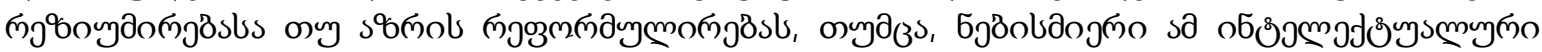

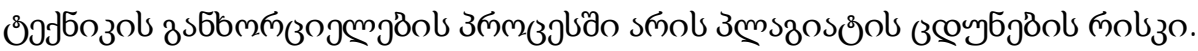

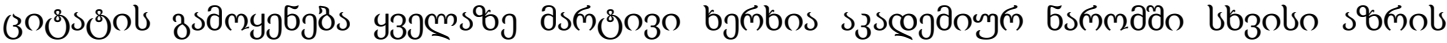

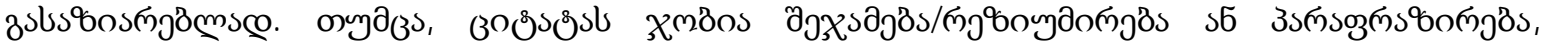

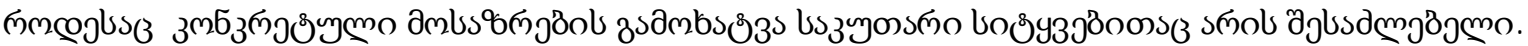

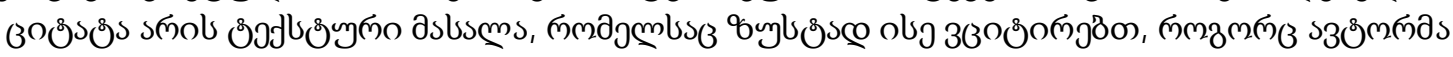

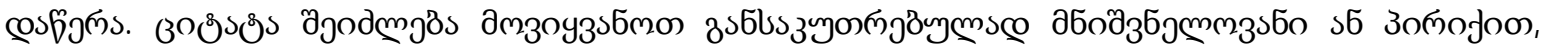

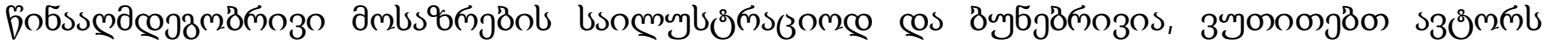

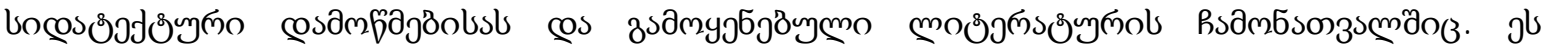

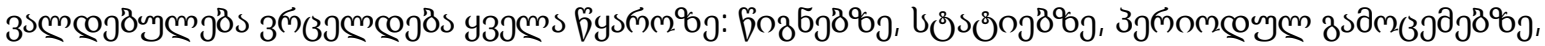

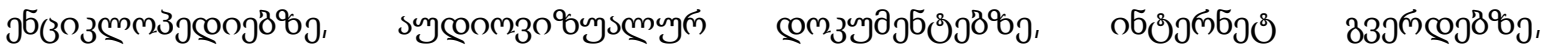

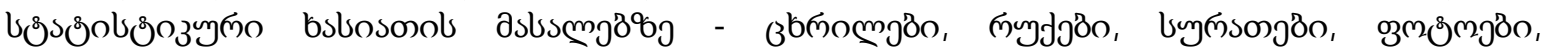

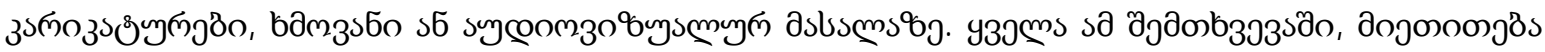

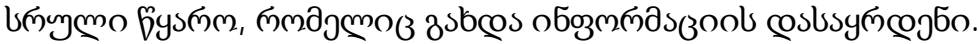

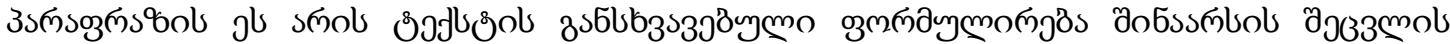

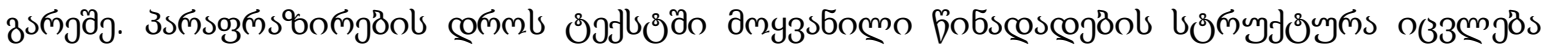

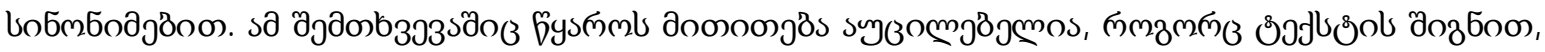

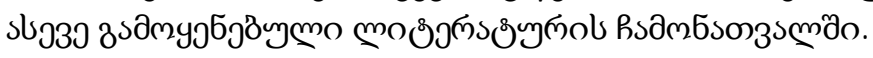

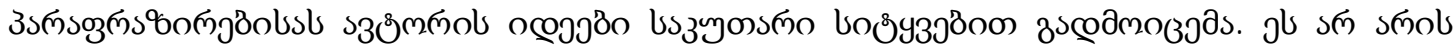

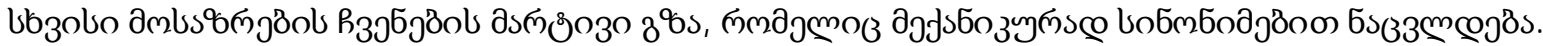

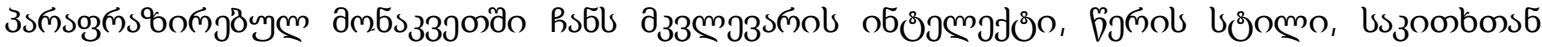

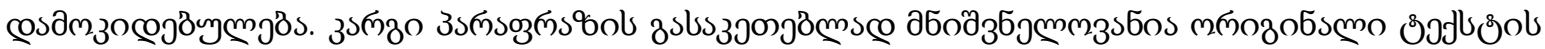

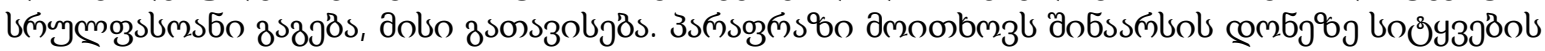

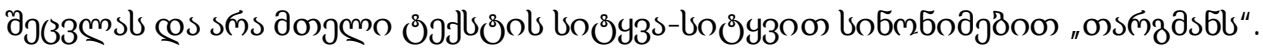




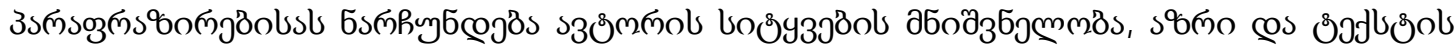

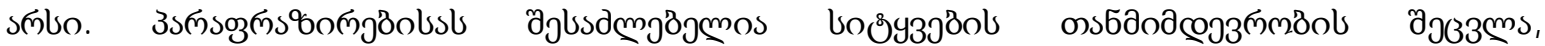

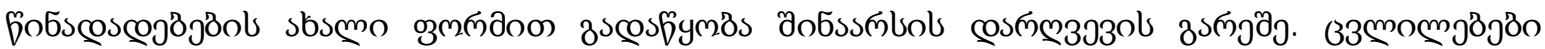

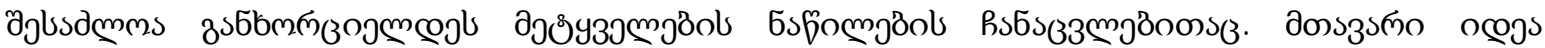

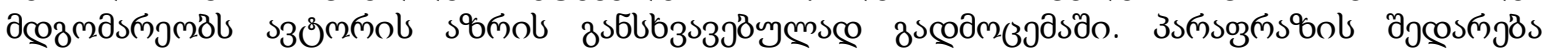

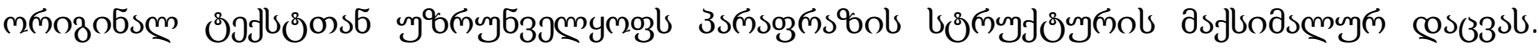

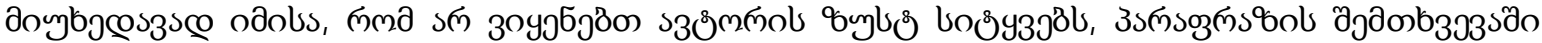

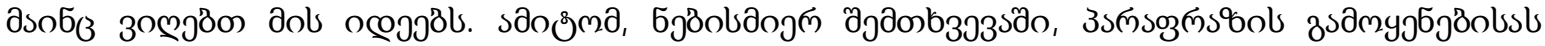

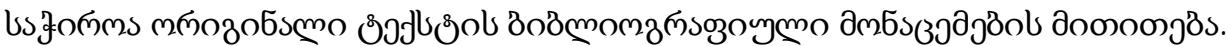

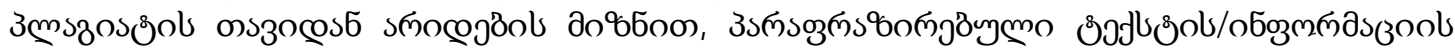

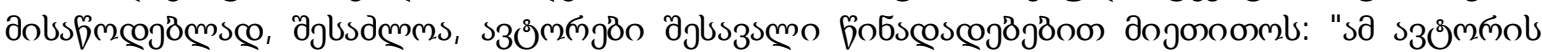

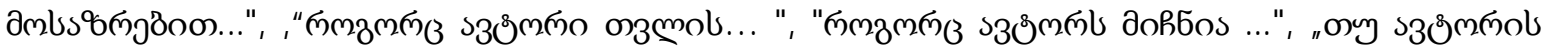

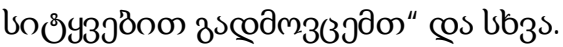

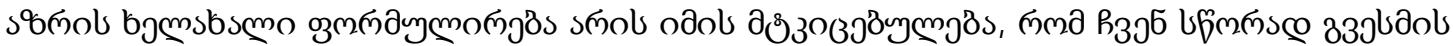

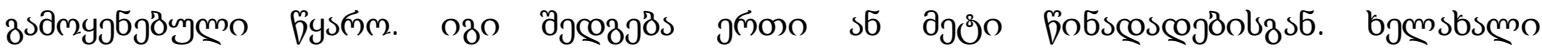

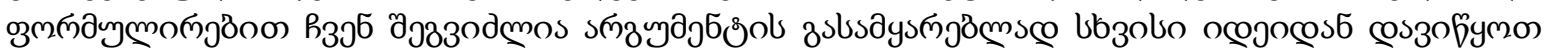

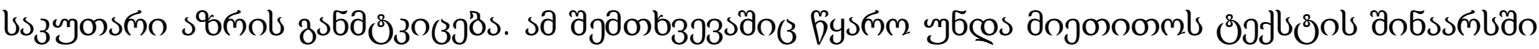

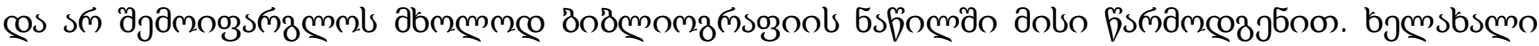

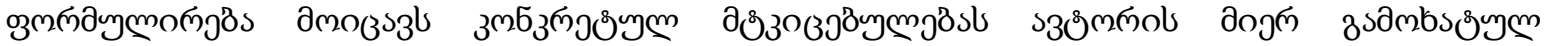

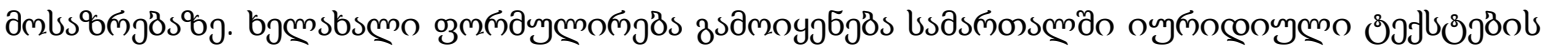

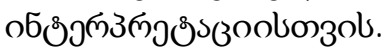

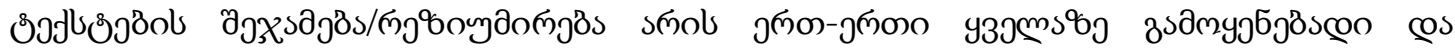

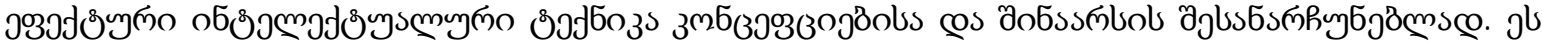

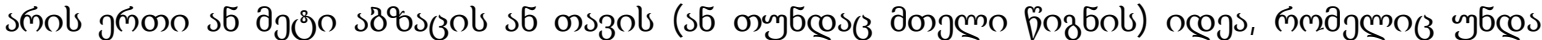

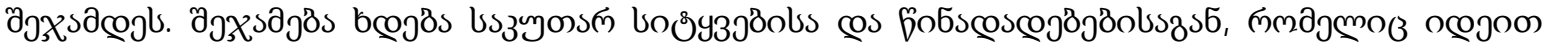

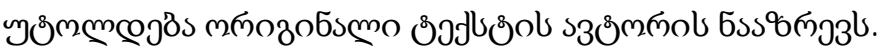

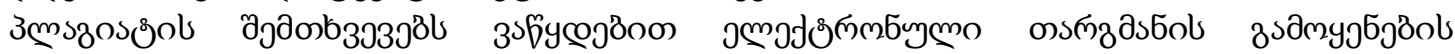

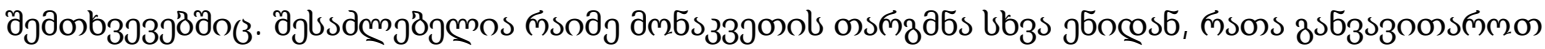

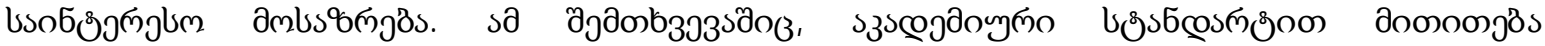

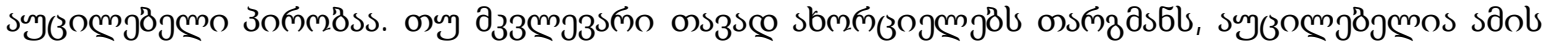

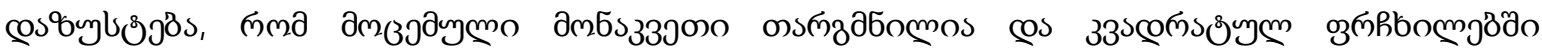

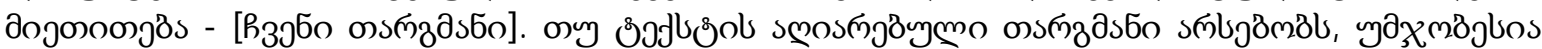

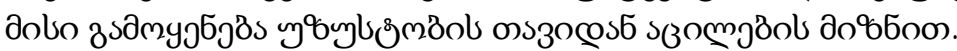

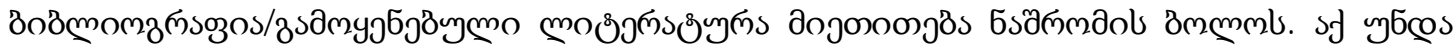

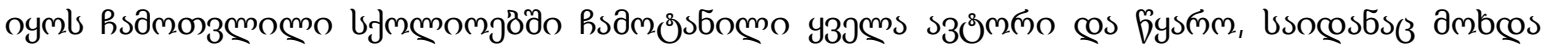

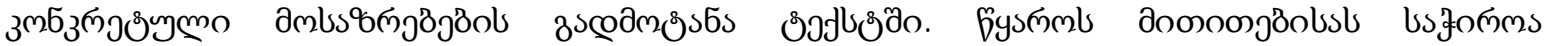

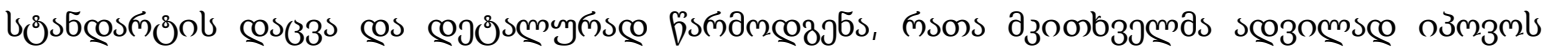

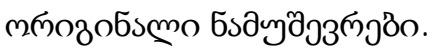

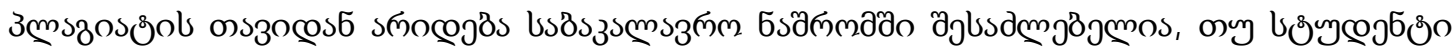

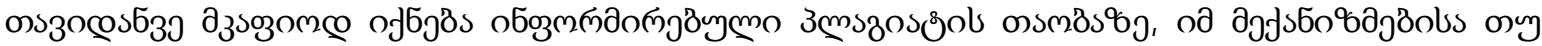

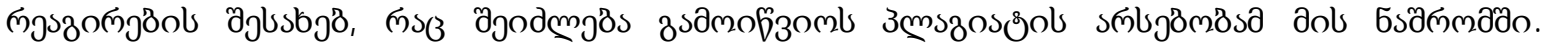

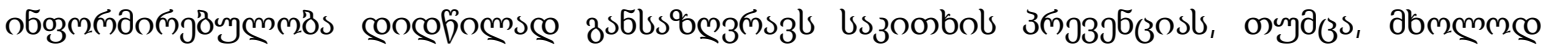

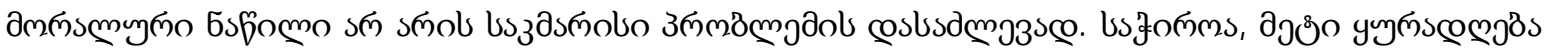

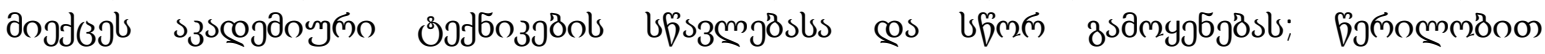

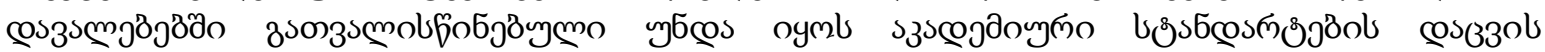
зизомлдемmдs.

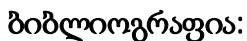




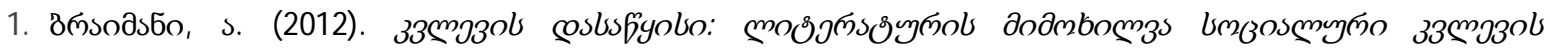

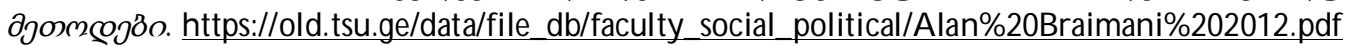

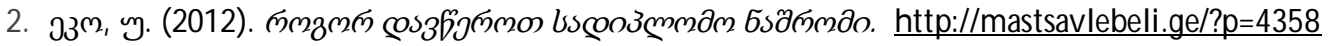

3. https://sites.uclouvain.be/infosphere boreal/fichiers communs/module7/paraphrase.html

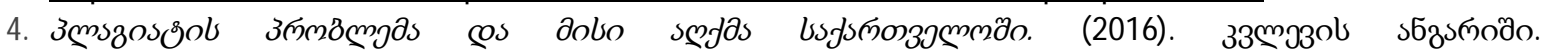
http://www.osgf.ge/files/2016/Publications/Plagiat___ge___2016.pdf

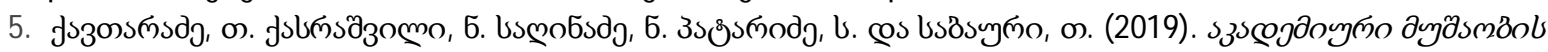

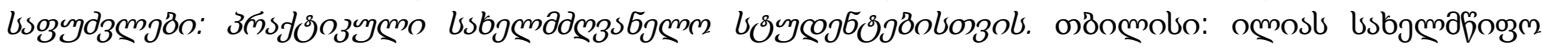

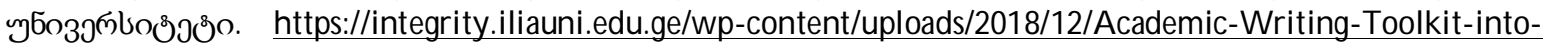
Georgian-akadmiuri-mushaobis-saphudzvlebi-praqtikuli-sakhelmdzghvanel o.pdf

\title{
Undergraduate Thesis and Plagiarism coping Strategies
}

Dzamukashvili Lali

Iakob Gogebashvili State University, Telavi

\begin{abstract}
Bachelor's degree is a final step before graduating first four years of studying at the university. The students show off their knowledge and research skills acquired by the University that are based on their original vision and scientific information. Writing a bachelor's thesis is a responsible, interesting and at the same time stressful job. It requires formal characteristics of its performance, which determine the content, form and style of the paper.

The researcher of the undergraduate thesis raises the problem, analyzes, substantiates and suggests the solutions, evaluates the research results and concludes. During the working process new knowledge is activated which is based on analytical skills and critical reasoning.

Maintaining academic standards in the paper makes it more fruitful because it gives the reader a sense that the author of the paper depends on reliable academic sources to support his or her own reasoning and position.

The completed paper will be checked for plagiarism. There are numerous programs which reveal the originality of the work. After all, there is a big temptation of plagiarism in the papers.

Preventing plagiarism offers to protect someone else's intellectual property and make the student aware that he or she has to respect the rights of intellectual property and distance himself or herself from plagiarism. Avoiding Plagiarism in the paper is possible if the student has been clearly informed about the mechanisms or responses that plagiarism may cause in his or her work. Awareness is largely responsible for preventing plagiarism, however, only the moral part of the problem is not enough. We need to pay more attention to proper implementation of the academic techniques in the academic environment.
\end{abstract}

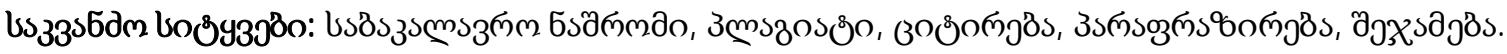

Keywords: Bachelor Thesis, Plagiarism, Citation, Paraphrasing, Summary. 